\title{
Treating Depression and Anxiety with Digital Cognitive Behavioural Therapy for Insomnia: A Real World NHS Evaluation Using Standardized Outcome Measures
}

\author{
Annemarie I. Luik \\ Sleep \& Circadian Neuroscience Institute, University of Oxford, and Big Health Ltd, London, UK
}

Sophie Bostock

Faculty of Medicine, University of Southampton, and Big Health Ltd, London, UK

Leanne Chisnall

Self Help Manchester, UK

Simon D. Kyle

Sleep \& Circadian Neuroscience Institute, University of Oxford, UK

Nicky Lidbetter and Nick Baldwin

Self Help Manchester, UK

Colin A. Espie

Sleep \& Circadian Neuroscience Institute, University of Oxford, and Big Health Ltd, London, UK

Background: Evidence suggests that insomnia may be an important therapeutic target to improve mental health. Aims: Evaluating changes in symptoms of depression and anxiety after supported digital cognitive behavioural therapy (dCBT) for insomnia delivered via a

Correspondence to Colin A. Espie, Sleep \& Circadian Neuroscience Institute, Nuffield Department of Clinical Neurosciences, University of Oxford, Sir William Dunn School of Pathology, South Parks Road, Oxford OX1 3RE, UK, and Big Health Ltd, 60-62 Commercial Street, London E1 6LT, UK. E-mail: colin.espie@ @ndcn.ox.ac.uk 
community-based provider (Self Help Manchester) of the Improving Access to Psychological Therapies (IAPT) service. Method: Supported dCBT for insomnia was delivered to 98 clients (mean age 44.9 years, $S D$ 15.2, 66\% female) of Self Help Manchester. All clients received six support calls from an eTherapy coordinator to support the self-help dCBT. During these calls levels of depression (Patient Health Questionnaire, PHQ-9) and anxiety (Generalized Anxiety Disorder, GAD-7) were determined. Results: Depression $\left(M_{\text {difference }}-5.7, t(70)=12.5, p<\right.$ $.001)$ and anxiety [Generalized Anxiety Disorder-7 (GAD-7), $M_{\text {difference }}-4.1, t(70)=8.0, p<$ $.001]$ were reduced following supported dCBT for insomnia. This translated into an IAPT recovery rate of $68 \%$ for depression and anxiety. Conclusions: These results suggest that dCBT for insomnia alleviates depression and anxiety in clients presenting with mental health complaints in routine healthcare.

Keywords: Depression, anxiety, insomnia, cognitive behavioural therapy, internet, online, computerized.

\section{Introduction}

In England, the Improving Access to Psychological Therapies (IAPT) programme of the UK National Health Services (NHS) aims to increase the availability of evidence-based mental health provision. Recently, sleep has been suggested as a novel therapeutic target to improve mental health (Christensen et al., 2016). Improving poor sleep might ameliorate insomnia symptoms that are shared with depression, but possibly also targets mechanisms that underlie depression. At the same time, digital Cognitive Behavioural Therapy (dCBT; via web and mobile) for insomnia has emerged as a scalable treatment with beneficial outcomes. In a randomized clinical trial, dCBT improved self-rated insomnia symptoms and sleep diary data (Espie at al., 2012). In this paper we evaluated changes in independent, standardized evaluations of depression and anxiety after dCBT for insomnia in an NHS-funded charity in the IAPT service and compared these outcomes to the IAPT target recovery rate $^{1}(\geq 50 \%)$.

\section{Method}

For this evaluation of a real-world clinical service, clients were enrolled from a communitybased provider of psychological therapies in Manchester, funded by the NHS (Manchester North, Central and South Clinical Commissioning Groups). Clients who complained of poor sleep in combination with self-reported symptoms of depression or anxiety between March 2014 and May 2015 were eligible for referral to supported dCBT for insomnia. Clients who were willing to access this form of treatment, received a free voucher code from the service's eTherapy coordinator.

The digital treatment (www.sleepio.com and associated Sleepio App) is fully automated and evidence-based in a placebo-controlled trial (Espie et al., 2012). It comprises six sessions lasting an average of 20 minutes. Its underlying algorithms feed the delivery of information, support, and self-help techniques in a personally tailored manner. Treatment content is based on CBT manuals and includes a behavioural component (sleep restriction, stimulus control, and relaxation), a cognitive component (paradoxical intention, cognitive restructuring, mindfulness, positive imagery, putting the day to rest) and an educational

\footnotetext{
${ }^{1}$ http://www.iapt.nhs.uk/silo/files/iapt-3-year-report.pdf
} 
component (psycho-education, sleep hygiene). The programme is highly interactive, and content is presented by an animated virtual therapist "The Prof". Participants complete daily sleep diaries throughout the intervention, which are used by the programme algorithmically to provide automated "personalized" help. In addition, participants complete a questionnaire at the beginning of therapy to set treatment goals. Throughout the course of therapy, participants have access to an online community and an online library of information about sleep. Participants can view their online "case file", which includes four sections: a progress review, a reminder of strategies to try out between sessions, an agreed sleep schedule, and a list of further reading.

In addition to the dCBT, the IAPT treatment protocol included six support calls between an eTherapy coordinator and client; these 20 to 30 minute calls are scheduled routinely to support the self-help treatment by signposting content within the programme, explaining techniques, giving suggestions on implementation, and determining treatment progress. The eTherapy coordinator, who does not require any formal psychological qualifications but receives on the job training within the service, did not provide additional CBT for insomnia content. No other IAPT interventions were accessed simultaneously. During these calls levels of depression and anxiety (via Patient Health Questionnaire (PHQ-9; Kroenke, Spitzer and Williams, 2001) and Generalized Anxiety Disorder (GAD-7; Spitzer, Kroenke, Williams and Löwe, 2006) respectively) were also determined. Insomnia was evaluated at baseline and the final assessment with the Insomnia Severity Index (ISI; Bastien, Vallières and Morin, 2001). Clients who do not improve may be referred to other treatments after the supported dCBT program. All data were independently gathered within the routine protocol of the IAPT service provider. Information on sex, age and the use of sleep medication was obtained within the dCBT program. The IAPT programme calculates recovery rates to evaluate their services (Gyani, Shafran, Layard and Clark, 2013).The "recovery rate" is the percentage of clients who score below the clinical cut-off on the PHQ-9 (>9) and GAD-7 (>7) after the treatment period over those who scored above the clinical cut-off on the PHQ-9 or GAD-7 before treatment. Those who move to recovery with a score change that exceeds the measurement error of the questionnaire are defined as having a "reliable recovery". T-tests (paired and unpaired) and linear mixed models were used to analyse the data (IBM SPSS version 22.0, IBM Corp., Somers, NY, USA). As this manuscript presents a service evaluation of a treatment within the IAPT programme no approval of a research ethics committee was obtained.

\section{Results}

A total of 120 clients received an access code for dCBT. In accordance with standard IAPT service evaluations (Gyani et al., 2013) persons who did not contribute data after baseline were excluded $(n=22)$. The average age of the remaining 98 clients was 44.4 years (SD 14.7), 66\% female and 14\% reported taking sleep medication. They scored an average of 11.0 (SD 4.9) on the PHQ-9, 8.6 (SD 5.3) on the GAD-7 and 18.5 (SD 3.7) on the ISI. In total, 71 clients $(72 \%)$ had moderate to severe depressive symptoms (PHQ-9 > 9) or moderate to severe anxiety symptoms (GAD-7 > 7) and 87 clients $(89 \%)$ experienced clinical insomnia (ISI > 14).

Of the 98 clients included in this evaluation, 72 finished the treatment (73\%). Another 15 clients completed between 4 and 6 sessions and 11 dropped out before session 4 . Completers of the dCBT for insomnia program differed significantly from non-completers in baseline 
measurements for depression $\left(M_{\text {completers }} 10.2, S D\right.$ 4.5, $M_{\text {non-completers }} 13.1, S D 5.3, t(96)=$ $-2.7, p=.009)$ and anxiety $\left(M_{\text {completers }} 7.8, S D 5.3, M_{\text {non-completers }} 10.6, S D 4.8, t(96)=-2.4\right.$, $p=.019)$.

Mean PHQ-9 was reduced from 10.1(SD 4.2) to 4.4 (SD 3.5) points $\left(M_{\text {difference }}-5.7\right.$, $t(70)=12.5, p<.001)$ and the mean anxiety score was reduced from $7.8(S D 5.1)$ to 3.7 $\left(S D\right.$ 3.6) points $\left(M_{\text {difference }}-4.1, t(70)=8.0, p<.001\right)$ in clients who completed measures at the end of the treatment period ( $n=71$; see Figure, panel A). A linear mixed model $(n=98$, unstructured) was used to account for missing data, the difference between before and after treatment remained significant (PHQ-9 $t(74)=13.7, p<.001$; GAD-7 $t(84)=9.4 p<.001$ ). Sex, age, and the intake of any sleep medication did not attenuate these associations.

The proportion of participants meeting "caseness" for depression and anxiety reduced (see Figure, panel B). Forty-eight (68\%) of the 71 clients who scored above the PHQ-9 or GAD7 threshold at baseline moved to recovery when the last observation was carried forward to ensure compatibility with national IAPT targets. When only complete cases were analysed the recovery rate was $58 \%$, such that $65 \%$ of the total population scored below case-level after the treatment period. In addition, 42 clients (59\%) had an IAPT defined reliable recovery, that is, persons who moved to recovery with $\geq 6$ point change on the PHQ-9 or $\geq 4$ point change on the GAD-7. Again, when only complete cases were analysed this rate was $52 \%$.

As expected, insomnia symptoms significantly decreased $\left(n=71, M_{\text {before }} 18.3, S D 3.5, M_{\text {after }}\right.$ $6.6, S D 4.5, t(70)=20.2, p<.001)$. Change in insomnia symptoms was significantly related to changes on the PHQ-9 $(r=0.32, p=.006)$ and GAD-7 $(r=0.41, p<.001)$.

\section{Discussion}

We report that targeted dCBT for insomnia delivered to clients accessing an IAPT service in the community confers significant benefit to mental health, consistent with findings from a recent efficacy trial (Christensen et al., 2016). Indeed, those who completed dCBT for insomnia demonstrated an IAPT recovery rate $(68 \%$, reliable recovery $59 \%)$, which is above the IAPT target of $\geq 50 \%$, and current IAPT reliable recovery rate of $43 \%{ }^{2}$. These findings are in keeping with contemporary data from sleep science that sleep disturbance is more than a symptom of psychological distress and that its explicit targeting may leverage mental health benefit (Christensen et al., 2016).

Digital CBT also substantially decreased insomnia symptoms per se. Moderate correlations between changes in insomnia and changes in depression and anxiety indicate improved sleep might serve as a mediator of outcome for depression and anxiety, but a trial will be required to test these associations in a robust way.

Limitations to our real-word evaluation include the lack of a treatment as usual comparison group or active control arm. However, we are encouraged by recent reports of face-to-face and web-based CBT for insomnia on mental health outcomes and, specific to England, treatment recovery rates comparable to national IAPT outcomes. In addition, on average, persons who did not complete the treatment had higher presenting scores on depression and anxiety. It may be that self-help programmes, at least in their current form, are best suited to patients with more moderate complaints. However, the number of participants who completed the dCBT

\footnotetext{
${ }^{2}$ March 2015, http://www.hscic.gov.uk/catalogue/PUB17755/IAPT-month-Mar-2015-exec-sum.pdf
} 
A
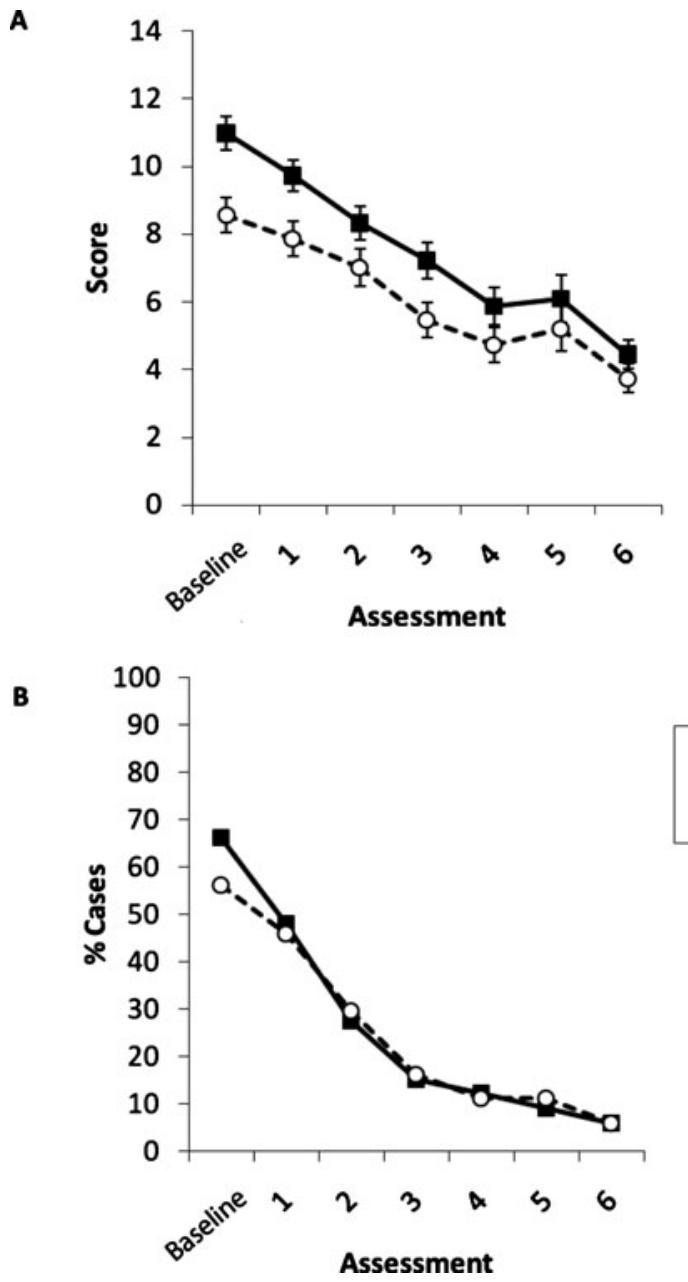

$\rightarrow-$ PHQ-9

$-\infty-G A D-7$ $\rightarrow-$ PHQ-9

$-\infty-G A D-7$

Figure 1. Change in depression and anxiety during treatment (number of observations per session: baseline $n=98$; assessment $1 n=93$; assessment $2 n=72$; assessment $3 n=63$; assessment $4 n=53$; assessment $5 n=39$; assessment $6 n=71$ ). Panel A reflects the change in score on the Patient Health Questionnaire (PHQ-9) and Generalized Anxiety Disorder (GAD-7); panel B reflects the percentage of persons who experience symptoms at a clinical level on the PHQ-9 and GAD-7.

treatment was $73 \%$, markedly higher than the current IAPT services average completion rate of $48 \%{ }^{2}$ which demonstrates the feasibility of a digital self-help program in the IAPT service.

In conclusion, this evaluation demonstrates a successful implementation of a digital selfhelp treatment for insomnia; treatment completion rate was above the IAPT average and recovery rates were substantially higher than other IAPT self-help treatments that directly target depressive and anxiety symptoms. 


\section{Acknowledgements}

Financial support: The current study represents a service evaluation of dCBT in the NHS IAPT programme. No specific funding for this evaluation was received.

Conflict of interests: The position of AIL is funded by Big Health Ltd, the company behind the digital CBT program evaluated in this study. SB is employed by Big Health Ltd. SDK has been a consultant for Sleepio, which is part of Big Health Ltd. CAE is the clinical and scientific director of Big Health Ltd and holds shares in Sleepio Ltd. LC and NB are employed by Self Help Manchester, NL is chief officer and founder of Self Help Manchester.

\section{References}

Bastien, C.H., Vallières, A. and Morin, C.M. (2001). Validation of the Insomnia Severity Index as an outcome measure for insomnia research. Sleep Medicine, 2, 297-307. doi:10.1016/S1389-9457(00)00065-4

Christensen, H., Batterham, P.J., Gosling, J.A., Ritterband, L.M., Griffiths, K.M., Thorndike, F.P., et al. (2016). Effectiveness of an online insomnia program (SHUTi) for prevention of depressive episodes (the GoodNight Study): a randomised controlled trial. Lancet Psychiatry, 3, 333-341. doi: 10.1016/S2215-0366(15)00536-2

Espie, C.A., Kyle, S.D., Williams, C., Ong, J.C., Douglas, N.J., Hames, P., et al. (2012). A randomized, placebo-controlled trial of online cognitive behavioral therapy for chronic insomnia disorder delivered via an automated media-rich web application. Sleep, 35, 769-781. doi: 10.5665/sleep. 1872

Gyani, A., Shafran, R., Layard, R. and Clark, D.M. (2013). Enhancing recovery rates: lessons from year one of IAPT. Behaviour, Research and Therapy, 51, 597-606. doi:10.1016/j.brat.2013.06.004

Kroenke, K., Spitzer, R.L. and Williams, J.B.W. (2001). The PHQ-9: validity of a brief depression severity measure. Journal of General Internal Medicine, 16, 606-613. doi:10.1046/j.1525-1497.2001.016009606.x

Spitzer, R.L., Kroenke, K., Williams, J.B.W. and Löwe, B. (2006). A brief measure for assessing generalized anxiety disorder: the GAD-7. Archives of Internal Medicine, 166, 1092-1097. doi:10.1001/archinte.166.10.1092 